Figure S1:

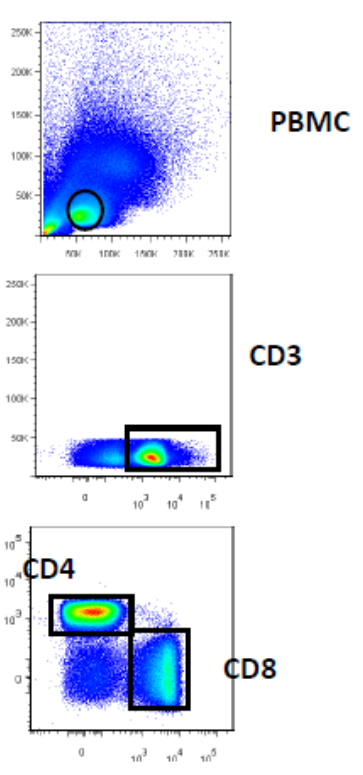

\title{
Gating strategy
}
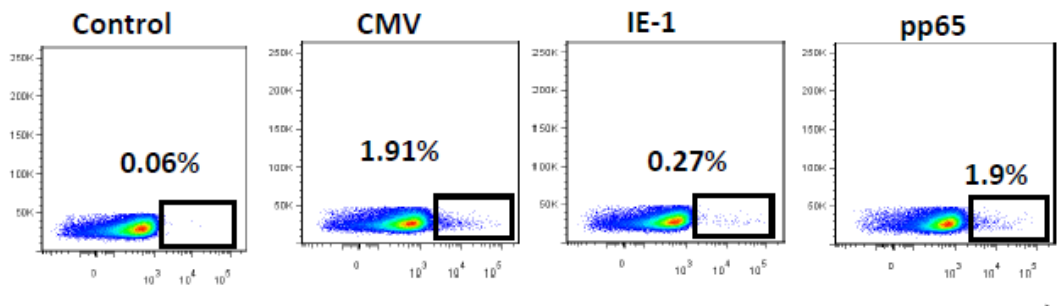

e.g. IFN- $\gamma$ 
Figure S2:
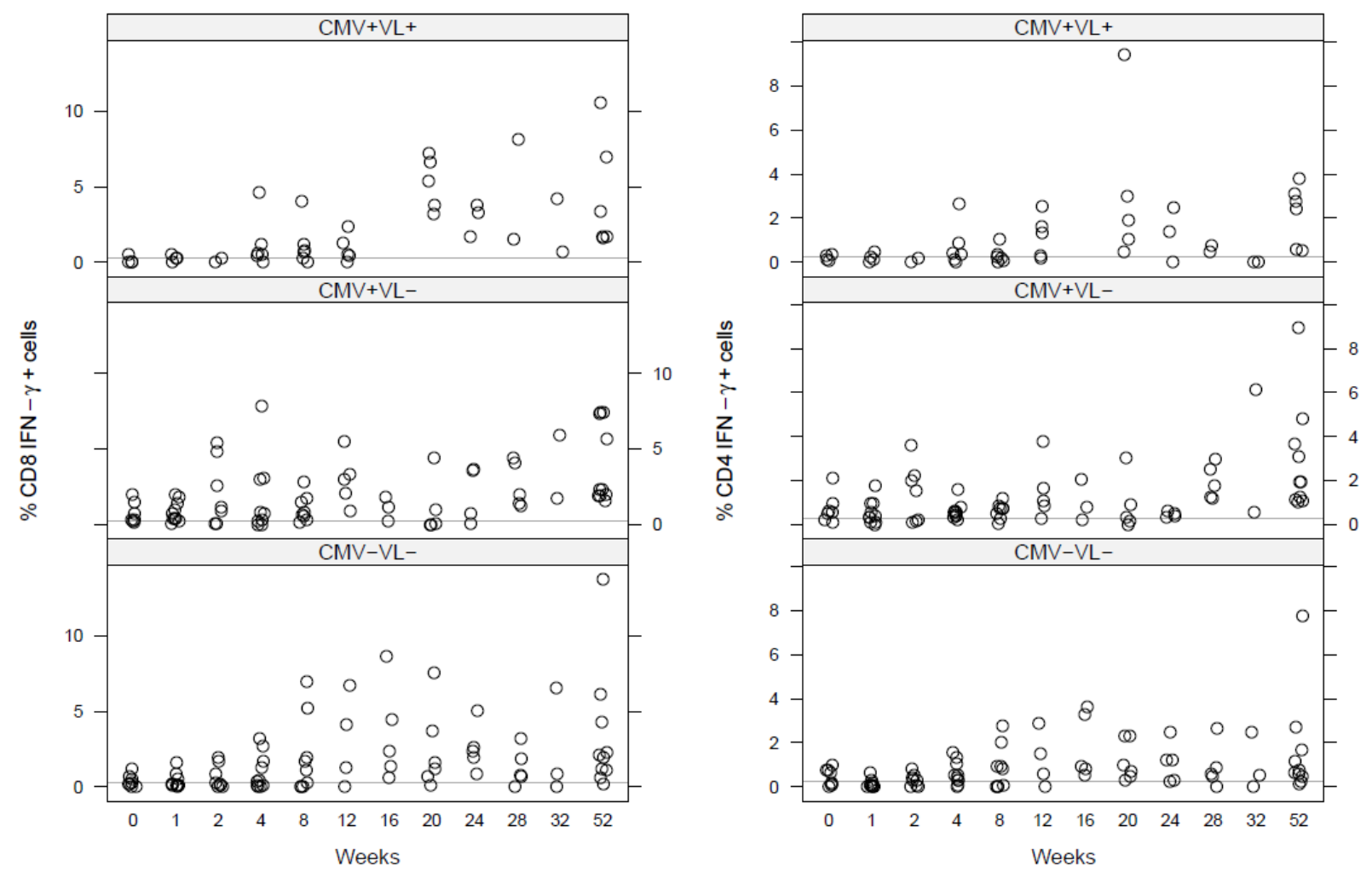


\section{Table S1}

\begin{tabular}{|c|c|c|c|c|c|c|c|c|c|c|c|c|c|c|c|c|c|c|c|c|c|}
\hline & \multicolumn{7}{|c|}{ CMV+VL+ } & \multicolumn{7}{|c|}{ CMV+VL- } & \multicolumn{7}{|c|}{ CMV-VL- } \\
\hline Week & $\underset{(\mathrm{pg} / \mathrm{mL})}{\text { IFN- })}$ & $\begin{array}{c}\text { TNF-a } \\
(\mathbf{p g} / \mathrm{mL})\end{array}$ & $\underset{(\mathrm{pg} / \mathrm{mL})}{\mathrm{IL}-2}$ & $\underset{(\mathrm{pg} / \mathrm{mL})}{\mathrm{IL}-15}$ & $\underset{(\mathrm{pg} / \mathrm{mL})}{\mathrm{TM}}$ & $\underset{(\mathrm{pg} / \mathrm{mL})}{\text { VEGF }}$ & $\underset{(\mathrm{pg} / \mathrm{mL})}{\mathrm{FKN}}$ & $\begin{array}{c}\text { IFN- }-\gamma \\
(\mathrm{pg} / \mathrm{mL})\end{array}$ & $\begin{array}{c}\text { TNF-a } \\
(\mathbf{p g} / \mathrm{mL})\end{array}$ & $\underset{(\mathrm{pg} / \mathrm{mL})}{\mathrm{IL}-2}$ & $\underset{(\mathrm{pg} / \mathrm{mL})}{\mathrm{IL}-15}$ & $\underset{(\mathbf{p g} / \mathbf{m L})}{\mathbf{T M}}$ & $\begin{array}{c}\text { VEGF } \\
(\mathrm{pg} / \mathrm{mL})\end{array}$ & $\underset{(\mathrm{pg} / \mathrm{mL})}{\mathrm{FKN}}$ & $\underset{(\mathrm{pg} / \mathrm{mL})}{\mathrm{IFN}-\gamma}$ & $\begin{array}{c}\text { TNF-a } \\
(\mathrm{pg} / \mathrm{mL})\end{array}$ & $\underset{(\mathrm{pg} / \mathrm{mL})}{\mathrm{IL}-2}$ & $\begin{array}{c}\text { IL-15 } \\
(\mathbf{p g} / \mathrm{mL})\end{array}$ & $\underset{(\mathbf{p g} / \mathbf{m L})}{\mathbf{T M}}$ & $\begin{array}{c}\text { VEGF } \\
(\mathrm{pg} / \mathrm{mL})\end{array}$ & $\underset{(\mathrm{pg} / \mathrm{mL})}{\mathrm{FKN}}$ \\
\hline 0 & 0.04 & 0.44 & 7.83 & 0.92 & 1611.2 & 24.2 & 1055.2 & 0.17 & 0.69 & 5.06 & 0.87 & 1131.7 & 100.5 & 1020.9 & 0.10 & 0.65 & 25.55 & 0.76 & 1422.1 & 41.5 & 724.6 \\
\hline 1 & 0.00 & 0.19 & 5.37 & 0.77 & 1381.0 & 66.7 & 977.5 & 0.11 & 0.63 & 3.92 & 1.98 & 1053.5 & 131.1 & 833.6 & 0.04 & 0.52 & 8.77 & 1.74 & 1523.7 & 78.4 & 711.0 \\
\hline 2 & 0.05 & 0.33 & 0.64 & 0.47 & 845.2 & 88.8 & 659.7 & 0.08 & 0.48 & 1.38 & 0.81 & 935.8 & 150.0 & 620.6 & 0.13 & 0.66 & 3.70 & 1.31 & 1240.7 & 85.4 & 922.1 \\
\hline 4 & 0.18 & 0.57 & 1.91 & 0.50 & 1308.2 & 97.0 & 587.5 & 0.15 & 0.65 & 1.38 & 0.73 & 1154.4 & 107.1 & 691.1 & 0.07 & 0.73 & 3.01 & 0.76 & 1322.6 & 98.0 & 778.1 \\
\hline 8 & 1.49 & 0.80 & 2.60 & 0.55 & 1774.2 & 49.4 & 776.3 & 0.25 & 0.67 & 0.82 & 0.82 & 1262.7 & 78.3 & 879.7 & 0.43 & 0.69 & 1.97 & 0.80 & 1272.4 & 81.5 & 626.3 \\
\hline 12 & 0.47 & 0.59 & 0.20 & 0.29 & 1620.5 & 45.6 & 760.8 & 0.38 & 0.68 & 0.31 & 0.67 & 1183.0 & 76.8 & 617.7 & 0.18 & 0.57 & 0.88 & 0.79 & 1827.5 & 43.0 & n.d. \\
\hline 16 & 0.29 & 0.54 & 4.75 & 0.30 & 1982.6 & 45.7 & 883.0 & 0.37 & 0.59 & 0.00 & 0.89 & 1239.6 & 38.7 & 603.8 & 0.11 & 0.36 & 0.26 & 0.39 & 1773.9 & 24.4 & 585.9 \\
\hline 20 & 0.33 & 0.78 & 0.60 & 0.07 & 1806.7 & 37.7 & 893.8 & 0.20 & 0.66 & 0.10 & 0.72 & 1101.3 & 64.4 & 577.2 & 0.37 & 0.54 & 0.63 & 0.59 & 1943.7 & 46.7 & 770.3 \\
\hline 24 & 2.06 & 1.29 & 0.63 & 0.10 & 3541.6 & 258.7 & 727.8 & 0.16 & 0.55 & 0.12 & 0.53 & 1444.6 & 48.8 & 694.6 & 0.18 & 0.54 & 0.45 & 0.69 & 1950.5 & 95.7 & 590.6 \\
\hline 28 & 0.43 & 0.93 & 2.81 & 0.63 & 1345.4 & 50.6 & 1600.6 & 0.27 & 0.57 & 0.10 & 0.49 & 1326.8 & 61.3 & 596.1 & 0.28 & 0.70 & 0.61 & 0.60 & 1992.9 & 28.5 & 958.7 \\
\hline 32 & 0.35 & 0.98 & 10.40 & 0.16 & 1319.6 & 110.8 & 750.8 & 0.39 & 0.78 & 0.21 & 0.72 & 1387.6 & 47.8 & 707.9 & 0.45 & 0.78 & 2.12 & 0.73 & 1876.3 & 41.9 & n.d. \\
\hline 36 & 0.55 & 1.36 & 0.74 & 0.05 & 3292.5 & 93.2 & 730.2 & 0.17 & 0.66 & 0.10 & 0.67 & 963.2 & 46.9 & 670.1 & 0.04 & 0.44 & 0.41 & 0.54 & 2405.1 & 42.1 & n.d. \\
\hline 40 & 0.05 & 0.21 & 0.17 & 0.07 & 1299.1 & 6.1 & 894.3 & 0.32 & 0.88 & 0.40 & 0.61 & 1156.9 & 235.9 & 651.6 & 0.48 & 0.74 & 0.85 & 0.73 & 2106.4 & 78.4 & 1446.2 \\
\hline 44 & n.d. & n.d. & n.d. & n.d. & n.d. & n.d. & n.d. & 0.42 & 0.64 & 0.00 & 0.78 & 1147.8 & 28.6 & 690.7 & 0.31 & 0.87 & 1.01 & 0.66 & 1952.5 & 44.4 & 1471.6 \\
\hline 48 & 0.01 & 0.93 & 0.53 & 0.12 & 3006.5 & 64.8 & 825.0 & 0.21 & 0.79 & 0.41 & 0.76 & 1573.8 & 69.3 & 762.5 & 0.03 & 0.62 & 0.51 & 0.63 & 1928.9 & 37.8 & n.d. \\
\hline 52 & 0.60 & 0.98 & 1.42 & 0.29 & 971.3 & 64.8 & 664.5 & 0.14 & 1.00 & 0.53 & 0.56 & 1094.7 & 72.3 & 848.7 & 0.40 & 0.78 & 0.59 & 0.69 & 1862.4 & 81.8 & 688.1 \\
\hline
\end{tabular}

IFN- $\mathrm{y}=$ interferon gamma, $\quad$ TNF- $\alpha=$ tumor necrosis factor alpha, $\quad$ IL-2 = Interleukin 2, IL-15 = Interleukin 15, $\quad$ TM

Thrombomodulin, $\quad$ VEGF = vascular endothelial growth factor,$\quad$ FKN $=$ CX3CL1/fractalkine, n.d.= not determined. 
SDC, Materials and Methods

\section{Detection of soluble biomarkers}

Plasma samples from were defrosted and used to measure VEGF, Thrombomodulin, IL2, TNF- $\alpha$, IFN- $y, I L-15$ and the atypical chemokine CX3CL1 (Fractalkine). Soluble biomarkers were detected by electrochemiluminescence using a MSD multi-array assays (Meso Scale Discovery; Rockville, MD, USA). For each biomarker, the plasma samples were diluted in the appropriate medium and the tests were performed according to manufacturer's instructions.

\section{Statistical model:}

GAMMs were used to model the temporal pattern in biomarker responses. Where the response variable was expressed as $0 / 1$ values (eg, the CD8-IFNy response to CMV lysate, pp65 or IE1 peptide greater or equal to $0.25 \%$ above control) a binomial distribution with logit link function was used. The proportion of the CD57+GB+population was modelled using a beta distribution with logit link function to handle the continuous and bounded (0-1) nature of the data. This model afforded considerably better fit to the observations and model diagnostics to the observations than a quasibinomial.

Smooth functions of the temporal pattern were fitted to the 3 patient groups via a smooth-factor interaction GAMM

$$
\mu_{i j}=g\left(\beta_{j} \text { PatientGroup }_{i}+s_{j}\left(\text { Week }_{i}\right)\right)^{-1}
$$




$$
Y_{i}=f_{\theta_{i}}\left(y_{i}\right)
$$

where $\mu_{i j}$ is the expectation of the response variable for the th patient in the patient group, $g()^{-1}$ is the inverse of the link function $g(), \beta_{j}$ represents the mean response for the $j$ th group and $s_{j}()$ is a separate smooth function of the number of weeks posttransplant representing the temporal trend for each patient group. $f_{\theta_{i}}$ indicates an extended exponential family distribution (binomial or beta) with parameters $\theta$ determined by $\mu_{i j .} y_{i}$ is an observation of the random variable $Y_{i}$.

To account for the repeated measurements on multiple patients, a random intercept term for Patient was introduced to the model above via a random effect smooth, which equates to an assumption of i.i.d. Gaussian random effects ${ }^{22}$.

Smoothness estimation for $s_{j}()$ was performed via the restricted maximum likelihood (REML) method with an additional penalty on the null space of each smooth. The additional penalty applies a form of shrinkage which allows for smooths to be penalized towards zero degrees of freedom and hence selected out of the model during fitting ${ }^{33}$.

To determine whether there were different responses in the 3 patient groups, models that included a single smooth for number of weeks posttransplant were also fitted. A likelihood ratio test was used to compare these nested models (refitted using maximum likelihood) to evaluate if patient group-specific time curves resulted in a better fit to the data than a single time curve for all 3 patient groups. 
All models were fitted using the mgcv package ${ }^{21-23}$ for the R statistical Software (version 3.2.2; $\left.{ }^{24}\right)$.

Table S1: Plasma levels of biomarkers

Figure S1: Analysis of PBMC populations and gating strategy

The density plot profiles of PBMCs are shown as examples of the selected cell populations of interest for the identification of IFN-ץ positive CD8+ and CD4+ T cells. 100 000 PBMC events were collected and selected. Cells were gated on $\mathrm{CD}^{+}$positive cells, and then on CD4+ and CD8+ positive cells. A region was set using the unstimulated control sample, and this area was then used to identify cell populations expressing surface markers CD4+ and CD8+ cells and within those cells producing IFN-y on samples stimulated with SEB, CMV lysate, IE-1 or pp65. For the analysis of cytokine production, a threshold of $0.25 \%$ above un-stimulated control was considered as positive.

Figure S2: Detection of IFN-y produced by CD8+ and CD4+ T cells of the 3 groups of patients stimulated with SEB

IFN- $y$ responses by CD8+ (left panel) and CD4+ (right panel) cells in response to Staphylococcus aureus enterotoxin B at the week of the transplant (W0), week 1, week 2, week 4, then at 4-weekly intervals until week 32, and a final sample at week 52. Upper panels show patients in the $\mathrm{CMV}+\mathrm{VL}+$ group, middle panels are the $\mathrm{CMV}+\mathrm{VL}$ - patient group, and the lower panel are patients in the CMV-VL- group. Each circle represents 1 
patient. A line has been drawn at $0.25 \%$ to illustrate where the positive response threshold was set. 